\title{
Multiple-Site Pain in Fibromyalgia- A Confirmatory Factor Analysis
}

\author{
Jussi Repo ${ }^{1}$, Mikhail Saltychev ${ }^{2}$ and Jari Ylinen ${ }^{3 *}$ \\ ${ }^{1}$ Department of Surgery, Central Hospital of Central Finland, Finland \\ ${ }^{2}$ Department of Physical and Rehabilitation Medicine, Turku University Hospital and University of Turku, Finland \\ ${ }^{3}$ Department of Physical Medicine and Rehabilitation, Central Hospital of Central Finland, Finland
}

Submission: February 1, 2018; Published: June 22, 2018

*Corresponding author: Jari Ylinen, Department of Physical Medicine and Rehabilitation Central Hospital of Central Finland, Keskussairaalantie 19, 40620 Jyväskylä, Finland, Email: jari.ylinen@ksshp.fi

\begin{abstract}
Fibromyalgia is a highly heterogeneous disorder occurring as widespread pain associated with general symptoms such as fatigue, depression, anxiety, insomnia, sexual dysfunction, and gastrointestinal conditions. This cross-sectional single tertiary medical center survey study aimed to investigate how multiple pain sites contribute to the construct of general pain in fibromyalgia. Altogether 266 women with fibromyalgia aged 18 to 60 years were included in the analysis. Confirmatory factor analysis of pain severity measured by a visual analogue scale ( 0 to 100 points) in seven major body regions. Headache, low back pain, and neck pain explained most of the total variance in general pain perceived across seven different body regions. Substantial correlations of $0.71,0.68$, and 0.61 were found between general pain and headache, low back pain, and neck pain, respectively. Pain reported for other four regions (chest, abdomen, upper extremity, and lower extremity) demonstrated fair correlations with general pain. When experiencing multi-site pain, women with fibromyalgia perceived headache, low back pain, and neck pain being subjectively more important than pain in other sites. While pain intensity and location commonly varies in fibromyalgia patients, it is important that one is aware of most common sites to be able to recognize fibromyalgia so that it can be managed properly.
\end{abstract}

Keywords: Fibromyalgia; Depression; Sexual dysfunction; Gastrointestinal conditions

\section{Introduction}

Fibromyalgia is a highly heterogeneous disorder occurring as widespread pain associated with fatigue, depression, anxiety, insomnia, sexual disfunction, and gastrointestinal conditions among other general symptoms [1-3]. Of all fibromyalgia symptoms, multi-site pain is the most characteristic one. It has been suggested that multi-site pain differs substantially by its origin and predictors from single-site pain. It has been proposed by several previous studies that multi-site pain, as well as its impact on functioning, can be better, or even solely, described by the number of sites involved instead of specific body pain sites [4-9]. In other words, according to previous knowledge, the absolute number of pain sites might be much more important than a particular pain distribution across different body regions. Previous studies have focused on evaluating the associations of pain sites' numbers and pain distributions with different scales and factors used mostly to describe patients' sociodemographic and health-related characteristics and the level of functioning [4-9]. However, the question remains - how patients themselves perceive the importance of pain experienced in different body regions? Does pain perceived in one particular region contribute as much to overall pain as pain in another site does? The answer may be of great interest when planning a treatment or developing new scales and surveys for patients with fibromyalgia and, probably, with other non-specific widespread pain conditions. The objective of the study was to investigate how different pain sites contribute to the construct of general pain in fibromyalgia.

\section{Methods}

\section{Setting and participants}

The participants were identified through a consecutive search on a hospital electronic patient record system between May 2007 and November 2015. The inclusion criteria were female gender, 18 to 60 years of age, and diagnosis 'M79' according the International Classification of Diseases, 10th Edition. The invitation to participate was sent to 1,042 patients. Of them, 286 patients agreed to participate in the study. They received a questionnaire with a prepaid return envelope. Pain severity was assessed in seven body sites (abdomen, chest, head, low back, neck, upper extremity, lower extremity) on a visual analogue scale 0 to 100 . The questionnaire included data on sociodemographic and occupational characteristics, comorbidities, medication, sleep quality, mood, alcohol and tobacco consumption, and the treatments received in the previous three months. 


\section{Statistical Analysis}

\section{Estimating the model}

The estimation procedure of Confirmatory Factor Analysis (CFA) employed the maximum likelihood method considering covariances supplied as input being unbiased. For simplicity, the estimates were reported in standardized form as correlation coefficients. A correlation $=<0.2$ was considered poor, from 0.21 to 0.4 fair, from 0.41 to 0.6 moderate, from 0.61 to 0.8 substantial, and $>0.8$ was considered perfect.

\section{Testing the goodness of model fit}

In order to assess how well the model matches the observed data, the root mean square error of approximation (RMSEA) was used as a main indicator. First, the model fit was tested assuming there were no covariances between unique factors
(Table 1). After that, the modification indices suggested by the software were used to add covariance between factors (doubleheaded arrows in Figure 1) one at a time, each time testing the RMSEA closeness to the value of $=<0.05$, or, at least, $=<0.08$ the threshold for accepting the model fit. Every insertion was considered plausible if it made logical sense and did not violate the assumption that the common and the unique factors are uncorrelated. After achieving the RMSE value of $=<0.05$, no further covariances were imputed and the goodness of fit was assessed using a chi-square test. As the sample was relatively small considering the requirements of CFA, in an attempt to reduce dependence on sample size, the relative (or 'normed') chi-square test was used. Relative chi-square is a chi-square estimate divided by the degrees of freedom. The chi square value $=<5.0$ was considered an indication of a good fit. There was no missing data in the analysis.

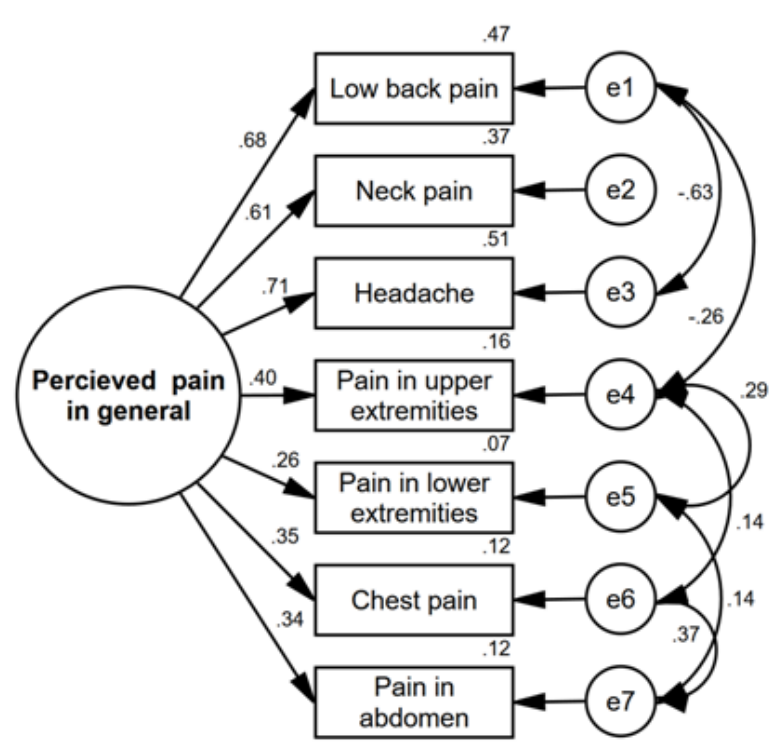

Figure 1: Confirmatory factor analysis. In the figure, circles represent unobserved and rectangles observed variables. 'e' variables represent a measurement error associated with the observed variable (variance that is not predicted by the latent factor). One-head arrows represent strength of correlation between two variables while two-head arrows strength of correlation between two covariant variables.

Table 1: Tests of the goodness of fit used.

\begin{tabular}{|c|c|c|c|}
\hline Method & Value & $90 \% \mathrm{CI}$ & CMIN/DF \\
\hline Goodness of fit index (GFI) & 0.994 & & \\
\hline Bentler-Bonett normed fit index (NFI) & 0.959 & & \\
\hline Bollen's relative fit index (RFI) & 0.894 & & \\
\hline Bollen's incremental fit index (IFI) & 0.994 & & \\
\hline Tucker-Lewis coefficient (TLI) & 0.983 & & \\
\hline Bentler's comparative fit index (CFI) & 0.994 & & \\
\hline Parsimony ratio (PRATIO) & 0.381 & & \\
\hline Parsimony adjustment to the NFI (PNFI) & 0.365 & & \\
\hline Parsimony adjustment to the CFI (PCFI) & 0.379 & & \\
\hline Akaike information criterion (AIC) & 63.3 & & \\
\hline
\end{tabular}


Journal of Yoga and Physiotherapy

\begin{tabular}{|c|c|c|c|c|}
\hline Browne-Cudeck criterion (BCC) & 65.6 & & & \\
\hline Hoelter's 'critical N' for a significance level of .05 (HOELTER .05) & 336 & & & \\
\hline Hoelter's 'critical N' for a significance level of .01 (HOELTER .01) & 435 & & & \\
\hline Method & Value & & & \\
\hline Noncentrality parameter (NCP) & 1.341 & 0 & 12.242 & \\
\hline Minimum value of the discrepancy (FMIN) & 0.046 & 0.007 & 0 & \\
\hline Root mean square error of approximation (RMSEA) & 0.029 & 0 & 0.0 .91 & \\
\hline Except for a constant scale factor (ECVI) & 0.314 & 0.307 & 0.372 & \\
\hline Method & Value & DF & p-value & \\
\hline Minimized value of the discrepancy function (CMIN) & 9.341 & 8 & 0.314 & 1.168 \\
\hline
\end{tabular}

All the analyses were conducted using IBM $₫$ SPSS $₫$ Statistics for Windows, Version 22.0, IBM Corp. Released 2013, Armonk, NY:USA IBM $®$ Corp.; IBM $®$ SPSS $® A$ Amos $^{\mathrm{TM}}$, Version 23.0, IBM® Corp. Released 2013, PA:USA IBM® Corp.; and Stata/ IC Statistical Software: Release 14. College Station (StataCorp LP, TX, USA).

\section{Results}

Of the 286 women, 266 returned the survey (response rate 93\%). The participants' average age was 47.2 (SD 10.8, range 19 to 61 ) years. The patients were slightly overweighed with body mass index on average 29.0 (SD 6.5, range 17.8 to 60.2 ) kg/ $\mathrm{cm}^{2}$. They reported a mean pain severity (summarized average for all body regions) of 40.1 (SD 15.7, range 4.8 to 82.1 ) points on a visual analogue scale. Detailed patients' sociodemographic, occupational and clinical characteristics are described in detail in Table 2. The confirmatory factor analysis model was built based on one latent factor - perceived general pain. Using modification indices suggested by the software, covariances were imputed resulting in the RMSEA declined to 0.029 (90\% CI 0.0 to 0.91 ) showing an acceptable fit. At this point, the relative Chi square value was 1.17 (below the cut-off point of 5.0) with 8 degrees of freedom. In other words, the model presented in Figure 1 demonstrated a good ability to describe the data observed in the study sample. Of the pain experienced in seven body areas, headache, low back pain, and neck pain explained most of the total variance within the common latent variablegeneral multi-site pain-demonstrating substantial correlations of $0.71,0.68$, and 0.61 , respectively. Pain reported for other body regions demonstrated only fair correlations.

Table 2: Participants' sociodemographic, occupational and clinical characteristics.

\begin{tabular}{|c|c|}
\hline Characteristic & $N=266$ \\
\hline \multicolumn{2}{|c|}{ Patient-Reported Comorbidities, n (\%) } \\
\hline Musculoskeletal & $132(49.6)$ \\
\hline Respiratory disease & $75(28.2)$ \\
\hline Neurological & $66(24.8)$ \\
\hline Heart or circulatory disease & $62(23.3)$ \\
\hline Mental disease & $44(16.5)$ \\
\hline Thyroid & $44(16.5)$ \\
\hline Allergy or hypersensitivity & $30(11.3)$ \\
\hline Diabetes & $21(7.9)$ \\
\hline Cancer disease & $7(2.6)$ \\
\hline Other & $151(56.8)$ \\
\hline \multicolumn{2}{|c|}{ Previous Operations, N (\%) } \\
\hline Musculoskeletal & $76(28.6)$ \\
\hline Gastrointestinal & $66(24.8)$ \\
\hline Urinary tract or reproductive organs & $48(18.1)$ \\
\hline Other & $168(63.2)$ \\
\hline Smokers, n (\%) & $114(42.9)$ \\
\hline Cigarettes per day, mean (SD) & $9.1(5.7)$ \\
\hline Alcohol consumption, n (\%) & $111(41.7)$ \\
\hline
\end{tabular}




\section{Journal of Yoga and Physiotherapy}

\begin{tabular}{|c|c|}
\hline Times per week, mean (SD) & $1.4(1.1)$ \\
\hline Units of alcohol per time, mean (SD) & $2.7(1.8)$ \\
\hline \multicolumn{2}{|c|}{ Income Source } \\
\hline Working & $111(41.7)$ \\
\hline Unemployment benefit & $48(18.0)$ \\
\hline Pension & $36(13.5)$ \\
\hline Sickness benefit & $15(5.6)$ \\
\hline Student benefit & $12(4.5)$ \\
\hline Partial pension & $11(4.1)$ \\
\hline Maternal/parental leave & $5(6.4)$ \\
\hline Alternation leave & $2(0.8)$ \\
\hline Other & $17(6.4)$ \\
\hline \multicolumn{2}{|c|}{ Treatment Visits in Previous 3 Months, Mean (SD) } \\
\hline Doctor & $1.2(1.7)$ \\
\hline Physiotherapist & $1.0(2.6)$ \\
\hline Massage therapist & $0.7(1.8)$ \\
\hline Naprapath & $0.0(0.4)$ \\
\hline Osteopath & $0.3(0.3)$ \\
\hline Chiropractor & $0.3(0.2)$ \\
\hline Other & $0.5(2.5)$ \\
\hline \multicolumn{2}{|c|}{ Therapies in Previous 3 Months, Mean (SD) } \\
\hline Stretch & $1.1(8.2)$ \\
\hline Electric & $0.0(0.4)$ \\
\hline Warmth & $0.3(0.3)$ \\
\hline Manipulations & $0.1(0.8)$ \\
\hline Cortisone injection & $0.1(0.4)$ \\
\hline Cold & $0.7(6.3)$ \\
\hline Acupuncture & $0.3(1.2)$ \\
\hline Massage & $0.8(2.0)$ \\
\hline Depression scale (DEPS), mean (SD) & $11.3(7.0)$ \\
\hline DEPS score>12 points, n (\%) & $92(34.6)$ \\
\hline Mood during the previous week, VAS $(0-100)^{*}$ & $30.44(22.5)$ \\
\hline Sleep quality during the previous week, VAS $(0-100)^{*}$ & $33.7(26.1)$ \\
\hline Pain medication, tablets during the previous week, n (\%) & $13.8(15.7)$ \\
\hline
\end{tabular}

$\mathrm{N}$ : Number of; SD: Standard Deviation; VAS: Visual Analogue Scale 0-100, from best possible to worst imaginable.

\section{Discussion}

This survey study evaluated the importance of different pain sites in perception of general pain experienced by 266 women with fibromyalgia. The patients perceived headache, low back pain, or neck pain more important than pain in other sites when experiencing widespread multi-site pain. The weaknesses of the study are its retrospective design and relatively small (for the purpose of confirmatory factor analysis) sample. The sample was limited to women only. Taking into account the modest size of the sample, the age range was probably too broad to generalize the construct structure of general pain across all age groups. However, this was the first study focusing on differences in importance of pain feelings in different body sites when there is widespread multi-site pain. The study employed the reliable method of confirmatory factor analysis and the size of sample was proved to be large enough to achieve good model fit and statistically significant results.

In Finnish population, 14\% of women are smoker [10]. In the present study, the rate of smokers was notably higher. Furthermore, in Finland, prevalence of depressive disorders 
in women is estimated at $10.0 \%$ and $4.3 \%$ of all cases are chronic [11]. In the present data $16.5 \%$ reported having a mental disorder and $34.6 \%$ of patients had a DEPS shore higher than 12 points indicating that those patients had at least $50 \%$ possibility for depression. Widespread pain risk factors have been described to be female sex, older age and at least two distressing somatic symptoms [4]. Furthermore, widespread pain can cause sickness absence more frequently than fewer pain sites [6,7]. Counting the pain sites has proposed to have value in management of fibromyalgia as it could give insight of the patients' general pain condition $[4,5]$. The present study suggests that not only the number of pain sites would be of importance in the management of patients with general pain but also how patients perceive pain in different body regions. There seems to be a hierarchy how different body regions contribute to general pain. Future prospects could include studies investigating the importance of pain location with risk factors, sickness absence and management of fibromyalgia.

Fibromyalgia is considered to be hereditary incurable pain syndrome. However, aerobic and therapeutic muscle strengthening and stretching exercises have shown to be effective way of reducing pain and sleep disturbance and increasing healthrelated quality of life $[12,13]$. Combining therapeutic massage with exercise seems to have an additional effect in improving pain, fatigue, sleep problems and depressive symptoms $[14,15]$. Drugs may be useful complement of an active rehabilitation program [16]. Also quitting smoking is an important part of treatment [17]. In conclusion, when experiencing multi-site pain, women with fibromyalgia perceived headache, low back pain, and neck pain being subjectively more important than pain in other sites. Patients often visit doctors and therapists complaining of pain only in these places. However, pain intensity and location commonly varies in fibromyalgia patients and thus it is important that doctors and therapists are aware of most common sites to be able to recognize fibromyalgia so that it can be managed properly.

\section{References}

1. Plazier M (2015) Pain characteristics in fibromyalgia: understanding the multiple dimensions of pain. Clin Rheumatol 34(4): 775-783.

2. Ablin JN, Häuser W (2016) Fibromyalgia syndrome: novel therapeutic targets. Pain Manag 6(4): 371-381.
3. Bazzichi L, Giacomelli C, Consensi A, Atzeni F, Batticciotto A, et al (2016) One year in review 2016: fibromyalgia. Clin Exp Rheumatol 34(2 Suppl 96): 145-149.

4. Coggon D, Ntani G, Palmer KT, Felli VE, Harari R, et al. (2013) Patterns of multisite pain and associations with risk factors. Pain 154(9): 17691777.

5. Kamaleri Y, Natvig B, Ihlebaek CM, Benth JS, Bruusgaard D (2008) Number of pain sites is associated with demographic, lifestyle, and health-related factors in the general population. Eur J Pain 12(6): 742748.

6. Haukka E, Kaila Kangas L, Ojajärvi A, Miranda H, Karppinen J, et al. (2013) Pain in multiple sites and sickness absence trajectories: a prospective study among Finns. Pain 154(2): 306-312.

7. De Cássia Pereira Fernandes R, Pataro SMS, de Carvalho RB, Burdorf A (2016) The concurrence of musculoskeletal pain and associated workrelated factors: a cross sectional study. BMC Public Health 16: 628.

8. De Fernandes RC, Burdorf A (2016) Associations of multisite pain with healthcare utilization, sickness absence and restrictions at work. Int Arch Occup Environ Health 89(7): 1039-1046.

9. Larsson B, Gerdle B, Björk J, Grimby Ekman A (2017) Pain Sensitivity and its relation to spreading on the body, intensity, frequency, and duration of pain: a cross-sectional population-based study (swepain). Clin J Pain 33(7): 579-587.

10. Helldán A, Helakorpi S (2015) Health behaviour and health among the finnish adult population, Spring 2014. National Institute for Health and Welfare (THL), Finland, p. 188.

11. Markkula N, Suvisaari J (2017) Prevalence, risk factors and prognosis of depressive disorders. Duodecim 133: 275-282.

12. Sosa-Reina MD, Nunez Nagy S, Gallego Izquierdo T, Pecos Martín D, Monserrat J, et al. (2017) Effectiveness of therapeutic exercise in fibromyalgia syndrome: a systematic review and meta-analysis of randomized clinical trials. Biomed Res Int 2017: 2356346.

13. Andrade A, Vilarino GT, Bevilacqua GG (2017) What is the effect of strength training on pain and sleep in patients with fibromyalgia? Am J Phys Med Rehabil 96(12): 889-893.

14. Toprak CS, Anaforoglu KB, Yasa ME, Sahbaz PC, Un Yildirim N, et al. (2017) A comparison of the effects of exercises plus connective tissue massage to exercises alone in women with fibromyalgia syndrome: a randomized controlled trial. Rheumatol Int 37(11): 1799-1806.

15. Yuan SL, Matsutani LA, Marques AP (2015) Effectiveness of different styles of massage therapy in fibromyalgia: a systematic review and meta-analysis. Man Ther 20(2): 257-264.

16. Kwiatek R (2017) Treatment of fibromyalgia. Aust Prescr 40(5): 179183.

17. Holloway BM, Santoro MS, Cronan TA (2017) Smoking, depression, \& stress: predictors of fibromyalgia health status. Psychol Health Med 22(1): 87-93. 
(c) (1) This work is licensed under Creative BY DOI: $10.19080 /$ JYP.2018.05.555663

\section{Your next submission with Juniper Publishers} will reach you the below assets

- Quality Editorial service

- Swift Peer Review

- Reprints availability

- E-prints Service

- Manuscript Podcast for convenient understanding

- Global attainment for your research

- Manuscript accessibility in different formats

( Pdf, E-pub, Full Text, Audio)

- Unceasing customer service

Track the below URL for one-step submission https://juniperpublishers.com/online-submission.php 